\title{
LHomme
}

L'HOMME Revue française d'anthropologie

212 | 2014

Varia

\section{Stanley Jeyaraja Tambiah (1929-2014)}

\section{Bernard Formoso et Justin Thomas McDaniel}

\section{OpenEdition \\ Journals}

Édition électronique

URL : http://journals.openedition.org/lhomme/23734

DOI : $10.4000 /$ lhomme.23734

ISSN : 1953-8103

\section{Éditeur}

Éditions de l'EHESS

\section{Édition imprimée}

Date de publication : 27 octobre 2014

Pagination : 63-70

ISSN : 0439-4216

\section{Référence électronique}

Bernard Formoso et Justin Thomas McDaniel, « Stanley Jeyaraja Tambiah (1929-2014) », L'Homme [En ligne], 212 | 2014, mis en ligne le 24 octobre 2016, consulté le 01 mai 2019. URL : http:// journals.openedition.org/lhomme/23734 ; DOI : 10.4000/lhomme.23734 


\title{
Stanley Jeyaraja Tambiah (|929-20|4)
}

\author{
Bernard Formoso \& Justin Thomas McDaniel
}

NE GRANDE FIGURE de l'anthropologie anglo-saxonne nous a quittés le 19 janvier 2014. Stanley Jeyaraja Tambiah, que ses collègues, étudiants et amis appelaient affectueusement "Tambi », a enseigné dans les départements d'anthropologie britanniques et américains les plus prestigieux. Assistant lecturer puis Fellow au King's College de Cambridge de 1964 à 1972, il fut ensuite professeur à l'Université de Chicago de 1973 à 1976, et occupa la chaire Esther \& Sidney Rabb de l'Université Harvard de 1976 jusqu'à son départ à la retraite en 2001. Son œuvre, riche en propositions théoriques marquantes, s'est déployée dans plusieurs directions : le bouddhisme theravada dans sa pratique populaire et sa dimension politique, la performativité des actes rituels, les classifications vernaculaires, l'histoire et le positionnement relatif dans la pensée occidentale des concepts de magie, de science et de religion, ou encore les conflits ethnonationalistes en Asie du Sud. Elle a exercé et exerce toujours une grande influence sur les chercheurs dont les travaux touchent à ces thématiques.

Jeyaraja Tambiah naquit dans le Ceylan de l'ère coloniale britannique, au sein d'une famille aisée de Tamouls chrétiens de Jaffna. Minoritaire parmi les minoritaires dans un pays où les Tamouls ne comptent que pour $18 \%$ de la population et sont hindouistes dans leur grande majorité, Tambiah se pensait, de surcroît, étranger dans son propre pays au sortir d'études secondaires où on lui avait enseigné l'histoire de la GrandeBretagne plutôt que celle de Ceylan et le latin plutôt que le cinghalais ou le tamoul ${ }^{1}$. Frustré par les cloisonnements communautaires et par le

1. Cf. un entretien réalisé le 8 juillet 1983 avec Alan MacFarlane, anthropologue et historien de l'Université de Cambridge, dont la vidéo est visible via le lien : http ://www.alanmacfarlane.com/ ancestors/Tambiah.html. 
déracinement culturel que le système colonial imposait aux élites locales, Tambiah suivit avec enthousiasme le cursus de sociologie-anthropologie proposé par l'Université de Ceylan à partir de 1948. Bryce Ryan, l'instigateur de ce cursus, fut son premier mentor en l'initiant aux enquêtes ethnographiques à la faveur de stages de terrain et en lui confiant, après l'obtention de son Bachelor of Art en 1951, un poste d'Assistant lecturer. Grâce à lui aussi, Tambiah obtint une bourse pour préparer, à partir de 1952, un doctorat en sociologie à l'Université Cornell aux États-Unis. Sous l'impulsion de Robin William, son directeur, il s'imprégna de la pensée de Talcott Parsons, Max Weber et Émile Durkheim. Soutenue en 1954, sa thèse, structurée autour des concepts de la sociologie rurale de Robert Redfield, avait pour titre The Process of Secularization in Three Ceylonese Peasant Communities. De retour à Ceylan en 1955, il réintégra l'Université de Ceylan et centra ses recherches sur l'analyse des structures de parenté en lien avec la tenure foncière dans divers villages du centre de l'île. En 1956, Tambiah fit une rencontre qui exerça une influence décisive sur le cours ultérieur de sa carrière : celle d'Edmund Leach qui complétait au même moment le recueil des matériaux ethnographiques pour son étude sur l'organisation sociale et la structure foncière du village de Pul Eliya. C'est en partie Leach qui, ayant dès le départ repéré son talent d'ethnologue et le fait qu'il travaillait sur des problématiques proches des siennes, lui obtiendra en 1963 les fellowships nécessaires pour venir le rejoindre et enseigner à Cambridge. Entre-temps, la situation des Tamouls au sein des milieux académiques cinghalais s'était dégradée, en raison de tensions interethniques qui allaient crescendo depuis 1956. Faute de pouvoir lever des fonds locaux pour conduire des recherches dans les zones reculées de la région tamoul avec son ami Gananath Obeyesekere, Tambiah chercha à s'expatrier et obtint un emploi d'expert en assistance technique de l'Unesco pour la Thaillande, rattaché au Bangkok Institute of Child Research. Ce poste, qu'il occupa entre 1960 et 1963, lui donna la possibilité de former sur le terrain des apprentis ethnologues thaïlandais, mais aussi de collecter la matière de son ouvrage Buddhism and the Spirit Cults in North-East Thailand (1970). Par l'intermédiaire de ce livre, il fut l'un des premiers auteurs à contester la dichotomie classiquement établie entre "grande» et "petite» tradition, entre les textes et leur mise en contexte. Le bouddhisme, dont pour sa part il décrivait la conceptualisation et la pratique populaire en milieu rural, mêlait étroitement finalités karmiques et intérêts immédiats (prospérité et fertilité des champs) en intégrant la religion de l'Illuminé et les cultes aux esprits en une commune orthopraxie. Plus tard, Tambiah confiera qu'il avait de longue date voulu comprendre le bouddhisme theravada et les rouages 
essentiels de son expansion, mais que, faute de pouvoir le faire dans son propre pays, il avait dû se rabattre sur d'autres terrains, la Thaïlande lui ayant offert cette opportunité 2 .

La venue de Stanley J. Tambiah, en 1963, à Cambridge scella la solide amitié et, surtout, la profonde affinité intellectuelle qui le liaient à Edmund Leach. Ces liens ressortent très bien du dernier ouvrage que Tambiah publia, Edmund Leach. An Anthropological Life (2002). Dans ce livre, il brosse un portrait attachant de la personnalité du célèbre anthropologue britannique, à partir de témoignages de ses anciens collègues et étudiants. Il y restitue en outre fidèlement l'apport de l'œuvre de Leach, ainsi que les débats théoriques, souvent pugnaces mais toujours dignes, qui marquèrent l'anthropologie britannique des années 19601970. À cette époque, Leach et Tambiah œuvrèrent de concert pour promouvoir, auprès des étudiants de Cambridge, les structuralismes de Roman Jakobson et de Claude Lévi-Strauss, mais aussi la cybernétique, la sémiotique et le travail de Gregory Bateson. Alors que leurs collègues de Cambridge, Meyer Fortes et Jack Goody, mettaient l'accent sur les règles de filiation à partir d'exemples africains, tous deux soulignaient l'importance des faits d'alliance à partir de matériaux sud ou sud-est asiatiques, et ambitionnaient de donner un nouveau souffle à l'anthropologie sociale britannique, en rupture avec le legs fonctionnaliste et structuralofonctionnaliste. Rangé au nombre des auteurs structuralistes par les recenseurs critiques de ses travaux, Tambiah était pourtant tout autant conscient de la fécondité que des limites de ce paradigme. Il dégageait, certes, des modèles de fonctionnement des sociétés ou des épures de leurs systèmes de classification, mais, en bon ethnographe, il s'appliquait aussi dans ses cours à les relativiser, voire à les déconstruire, en leur opposant de prosaïques contingences économiques et politiques, des anomalies historiques ou des contradictions cognitives. Il était aussi un éloquent critique d'autres structuralistes, dont Melford Spiro. Fervent admirateur de la philosophie analytique de John Austin, il était particulièrement fier d'avoir mis en évidence la dimension performative des actes rituels, notamment dans son recueil d'essais, Culture, Thought, and Social Action (1985).

World Conqueror \& World Renouncer, paru en 1976, confirma définitivement Tambiah dans son statut de grand spécialiste du bouddhisme theravada et de la société thaïe. Dans ce livre, il élargissait la perspective au-delà de l'approche monographique qu'il avait adoptée dans Buddhism and Spirit Cults, pour aborder de grands thèmes, tels que l'organisation des royaumes indianisés, le bouddhisme, l'institution monastique, 
le brahmanisme et le tantrisme. Il espérait produire une synthèse à la fois historique et ethnologique pour trouver un écho le plus large possible auprès des spécialistes d'autres disciplines (politistes, philologues, indianistes) - et, ainsi, justifier son titre de professeur au sein de prestigieuses universités américaines (Chicago, puis Harvard) qui recrutaient alors bien peu de chercheurs issus des pays du «tiers-monde». Il atteignit pleinement cet objectif au prix d'un énorme travail de compilation de sources éparses relatives aux textes du canon bouddhique et à l'histoire des royautés d'Asie du Sud-Est. En dépit du contenu très dense de World Conqueror \& World Renouncer, Tambiah y exposait clairement un modèle galactique d'organisation des royautés sud-est asiatiques prémodernes, valable pour le Siam mais aussi pour les autres sociétés à État de la région. Il y dégageait la structure générale de ce modèle en comparant plusieurs déclinaisons socioculturelles et historiques. Il mettait en relief son ancrage profond dans une idéologie et une ritualité du pouvoir d'origine indienne, qui campent le souverain en pivot de l'ordre sociocosmique. Mais, belle illustration de son structuralisme tempéré, il rendait compte aussi de la fragilité et donc du dynamisme interne des systèmes politiques élaborés de la sorte. Enfin, il démontrait en quoi la légitimité du souverain bouddhique se nourrit de l'idéal ascétique incarné par le clergé. Plus tard, dans un troisième ouvrage, The Buddhist Saints of the Forest and the Cult of Amulets (1984), Tambiah ira plus loin dans l'analyse des rapports étroits qui lient, en Thaïlande, l'institution monarchique et le clergé bouddhique, en montrant comment les monarques de l'époque moderne ont instrumentalisé les ascètes les plus fameux pour capter leur prestige et exalter le nationalisme thaï. Dans cet ouvrage, il examinait aussi, par référence aux diverses acceptions wébériennes de la notion de charisme, le pouvoir surnaturel dont les arahants ${ }^{3}$ thaïs sont investis et le processus de fétichisation mercantile dont ils font l'objet via le commerce des amulettes. Ce faisant, il articula dans une même problématique les usages politiques et populaires du bouddhisme, qu'il avait appréhendés séparément dans ses deux précédentes études. Ces trois ouvrages restent des références incontournables pour quiconque étudie le bouddhisme en Asie du Sud-Est. Dans leur complémentarité, ils illustrent parfaitement le projet scientifique de Tambiah, qu'il définissait ainsi en 1987 :

«Pour le meilleur ou pour le pire, je considère mes travaux sur le bouddhisme, la société et l'organisation politique [en Thaïlande] comme situés à la confluence de l'anthropologie, de l'indologie (études bouddhistes et hindouistes), et de l'histoire [...]. Le défi [...] fut de trouver ma voie en direction d'une anthropologie historique nourrie des acquis de l'indologie" (1987b : 187-188).

3. Moines bouddhistes devenus des saints pendant leur vie ou après leur mort. 
En 1983, alors qu'il lui restait des centaines de pages de notes issues de ses terrains thailandais et dont il aurait encore pu tirer un grand parti scientifique, Stanley J. Tambiah ressentit le besoin de réorienter ses recherches vers son pays d'origine, renommé Sri Lanka depuis 1972. Il vivait, en effet, comme un traumatisme personnel le conflit interethnique qui opposait les Tamouls et les Cinghalais, et qui prenait alors de plus en plus d'ampleur. Selon ses propres dires, analyser les racines de cette guerre civile eut pour lui l'effet d'une thérapie. Sa première contribution en ce sens fut Sri Lanka. Ethnic Fratricide and the Dismantling of Democracy (1986). Dans ce livre, il passait en revue les facteurs socio-économiques et les mesures gouvernementales, en matière éducative et linguistique notamment, qui, combinés, avaient engendré la crise. À la suite de ce diagnostic d'ensemble, il étudia, dans un autre ouvrage, l'histoire des moines activistes cinghalais tout en analysant les causes de leur engagement croissant dans la défense des intérêts des « fils du sol » cinghalais face aux " envahisseurs" tamouls hindouistes. Il leva aussi le voile sur leur lourde responsabilité dans le déclenchement des violences intercommunautaires, en contradiction avec les valeurs bouddhistes de tolérance, de détachement et de non-violence qu'ils ont pourtant pour rôle de promouvoir. L'étude fut publiée sous le titre Buddhism Betrayed? Religion, Politics, and Violence in Sri Lanka (1992) ${ }^{4}$. Dès sa parution, elle fit l'objet de vives critiques de la part d'intellectuels nationalistes cinghalais qui voulurent en interdire la publication au Sri Lanka. Ces attaques partisanes, qui le présentaient comme un ennemi de sa patrie d'origine, affectèrent beaucoup Tambiah. Il surmonta néanmoins l'épreuve en produisant un troisième livre sur le sujet, moins centré sur Sri Lanka et à l'ambition théorique plus ouvertement affichée : il y comparait les violences interethniques au Sri Lanka, en Inde et au Pakistan, pour produire une grille d'analyse sophistiquée ${ }^{5}$ des processus sociologiques étant au principe de la formation des foules émeutières et des violences collectives à caractère ethnique. Cet ouvrage, Leveling Crowds (1996), lui valut le prestigieux prix Balzan, en 1997. La même année, le Royal Anthropological Institute of Great Britain and Ireland lui décerna le Huxley Memorial Medal and Lecture. D’autres distinctions se succédèrent les années suivantes. Elles ne purent cependant

4. Cf. le compte rendu de cet ouvrage par Bernard Formoso, paru dans L'Homme, 1992, 131 : 198-201.

5. Cette grille d'analyse reposait sur les concepts de nationalization/parochialization pour rendre compte des rapports entre le centre du pouvoir et la périphérie contestataire, sur ceux de focalization/ transvaluation permettant, inversement, de rendre compte des mobilisations opérant à partir de la périphérie en direction du centre et, enfin, sur les concepts de routinization/ritualization pour décrire les modes opératoires de la violence collective. 
compenser le mal-être, particulièrement perceptible au cours des dernières années de sa vie, que suscitait en lui le rejet dont il avait fait l'objet dans son pays d'origine après la publication de ses analyses, pourtant pénétrantes, des racines du conflit sri-lankais.

Stanley J. Tambiah s'affirma au fil de sa carrière comme un grand ethnologue, l'un des meilleurs spécialistes de l'Asie du Sud-Est et du Sud, mais aussi comme un brillant philosophe des sciences et historien des idées. Sa grande contribution en ce sens fut la Lewis Henry Morgan Lecture qu'il donna à l'Université de Rochester, en 1984, qui parut dans une version augmentée en 1990, sous le titre Magic, Science, Religion, and the Scope of Rationality. Dans ce livre, que tout étudiant en ethnologie devrait placer parmi ses lectures prioritaires, il passe au crible de l'analyse comparée les thèses de grands auteurs (Tylor, Frazer, Malinowski, LevyBruhl, Wittgenstein, Weber, Elster ou Foucault) à propos des rapports entre magie et rationalité. Cette analyse débouche sur d'utiles développements concernant les critères universaux de la rationalité, le relativisme, les problèmes soulevés par la traduction et la commensurabilité des faits culturels, ou encore l'impact des inférences externes sur les régimes de scientificité.

Ses étudiants de Cambridge et de Harvard gardent de Stanley J. Tambiah le souvenir d'un enseignant brillant, donnant des cours clairs et structurés, et ne comptant pas son temps pour leur prodiguer des conseils personnalisés, les accompagner dans leurs projets ou, tout simplement, leur insuffler, au fil de conversations informelles, sa passion contagieuse pour l'ethnologie 6 . Pour beaucoup de ceux qui sont devenus des grands noms dans le domaine des études asiatiques, il incarna une figure tutélaire bienveillante et proche, en même temps qu'un formidable inspirateur d'émulation scientifique.

Université Paris Ouest-Nanterre-La Défense Département d'ethnologie, Nanterre bernard.formoso@mae.u-paris10.fr

University of Pennsylvania

Department of Religious Studies, Philadelphia (États-Unis) jmcdan@sas.upenn.edu

6. Cf., notamment, l'hommage rendu par certains de ses anciens étudiants de Cambridge et Harvard, consultable sur : http ://www.socanth.cam.ac.uk/2014/01/cambridge-social-anthropologyremembers-s-j-tambiah/. 


\section{Tambiah, Stanley Jeyaraja}

1957 The Disintegrating Village. Report of a Socio-Economic Survey. Colombo, Ceylon University Press Board (avec N. K. Sarkar). 1958 "The Structure of Kinship and its Relationship to Land Possession and Residence in Pata Dumbara, Central Ceylon ", Journal of the Royal Anthropological Institute of Great Britain and Ireland 88 (1) : 21-44.

1963 "Ceylon", in Bert F. Hoselitz \& Richard D. Lambert, eds, The Role of Savings and Wealth in Southern Asia and the West. Paris, Unesco : 44-125.

1966 "Polyandry in Ceylon ", in Christoph von Fürer-Haimendorf, ed., Caste and Kin in Nepal, India, and Ceylon. BombayNew York, Asia Publishing House : 264-358. 1967 "The Politics of Language in India and Ceylon ", Modern Asian Study 1 (3) : 215-240.

1968a "The Magical Power of Words ", Man 3 (2) : 175-208.

1968 b "Literacy in a Buddhist Village in North-East Thailand ", in Jack Goody, ed., Literacy in Traditional Societies. Cambridge, Cambridge University Press : 85-131.

1968c «The Ideology of Merit and the Social Correlates of Buddhism in a Thai Village", in Edmund R. Leach, ed., Dialectic in Practical Religion. London, Department of Archaeology and Anthropology by Cambridge University Press («Cambridge Papers in Social Anthropology " 5) : 41-121. 1969 "Animals Are Good to Think and Good to Prohibit ", Ethnology 8 (4) : 423-459.

1970 Buddhism and the Spirit Cults in North-East Thailand. Cambridge, Cambridge University Press.

1973a «From Varna to Caste Through Mixed Unions ", in Jack Goody, ed., The Character of Kinship. Cambridge, Cambridge University Press : 191-230. 1973b «Buddhism and This-Worldly

Activity ", Modern Asian Studies 7 (1) : 1-20.

1973c «The Persistence and

Transformation of Tradition in Southeast Asia, with Special Reference to Thailand", Dedalus 102 (1) : 55-84.

1973d "Dowry and Bridewealth and the Property Rights of Women in South Asia ", in Jack Goody \& Stanley J. Tambiah, eds, Bridewealth and Dowry. Cambridge,

Cambridge University Press

("Cambridge Papers in Social

Anthropology » 7) : 59-169.

1973e «Classification of Animals in Thailand ", in Mary Douglas, ed., Rules and Meanings. The Anthropology of Everyday Knowledge. Selected Readings. Oxon-New York, Routledge : 127-166.

1976 World Conqueror \& World Renouncer. A Study of Buddhism and Polity in Thailand against a Historical Background. CambridgeNew York, Cambridge University Press.

1977a «The Galactic Polity: The Structure of Traditional Kingdoms in Southeast Asia ", Annals of the New York Academy of Sciences 293 : 69-97.

1977b «The Cosmological and Performative Significance of a Thai Cult of Healing through Meditation ", Culture, Medicine and Psychiatry 1 (1) : 97-132.

1981 «A Performative Approach to Ritual ", Proceedings of the British Academy 65: 113-169.

1983 "On Flying Witches and Flying Canoes: The Coding of Male and Female Values ", in Edmund Leach \& Jerry W. Leach, eds, The Kula. New Perspectives on Massim Exchange. Cambridge, Cambridge University Press : 171-200. 1984 The Buddhist Saints of the Forest and the Cult of Amulets. A Study in Charisma, Hagiography, Sectarianism, and Millennial Buddhism. Cambridge, Cambridge University Press ("Cambridge Studies in Social Anthropology " 49). 
1985 Culture, Thought and Social Action. An Anthropological Perspective. Cambridge, Cambridge University Press.

1986 Sri Lanka. Ethnic Fratricide and the Dismantling of Democracy. Chicago, University of Chicago Press.

1987a The Buddhist Conception of Universal King and Its Manifestations in South and Southeast Asia. Kuala Lumpur, University of Malaya.

$1987 \mathrm{~b}$ «At the Confluence of Anthropology, History and Indology ", Contributions to Indian Sociology 21 (1) : 187-216.

1988 «Ethnic Fratricide in Sri Lanka : An Update ", in Remo Guidieri, Francesco Pellizzi \& Stanley J. Tambiah, eds, Ethnicities and Nations. Processes of Inter-Ethnic Relations in Latin America, Southeast Asia, and the Pacific. Austin, Rothko Chapel : 293-319.

1989a «Ethnic Conflict in the World Today ", American Ethnologist 16 (2) : 335-349.

1989b «Bridewealth and Dowry Revisited: The Position of Women in Sub-Saharan
Africa and North India ", Current Anthropology 30 (4) : 413-435.

1990a "Presidential Address : Reflexions on Communal Violence in South Asia", Journal of Asian Studies 49 (4) : 741-760.

1990b Magic, Science, Religion, and the Scope of Rationality. CambridgeNew York, Cambridge University Press («The Lewis Henry Morgan Lectures» 1984).

1992 Buddhism Betrayed? Religion,

Politics, and Violence in Sri Lanka.

Chicago, University of Chicago Press.

1996 Leveling Crowds. Ethnonationalist Conflicts and Collective Violence in South Asia. Berkeley, University of California Press ("Comparative Studies in Religion and Society"10).

1998 "What Did Bernier Actually Say? Profiling the Mughal Empire ", Contributions to Indian Sociology 32 (2) : 361-386.

2002 Edmund Leach. An Anthropological Life. Cambridge-New York, Cambridge University Press. 